Macrae, C. Investigating for improvement? Five strategies to ensure national patient safety investigations improve patient safety. Journal of the Royal Society of Medicine, 112(9), 365369. Copyright @ 2017 Carl Macrae. DOI: https://doi.org/10.1177/0141076819848114

\title{
Investigating for improvement? Five strategies to ensure national patient safety investigations improve patient safety
}

Short title: Investigating for Improvement?

Carl Macrae PhD

Centre for Health Innovation, Leadership and Learning, Nottingham University Business School, University of Nottingham, Jubilee Campus, Wollaton Road, Nottingham, NG8 1BB

Correspondence to:

Carl Macrae, Nottingham University Business School, University of Nottingham, Jubilee Campus, Wollaton Road, Nottingham, NG8 1BB, UK

\section{carlmacrae@mac.com}

Competing interests: CM acted as Specialist Advisor to the Public Administration Select Committee on investigating incidents in healthcare, served on the Healthcare Safety Investigation Branch (HSIB) Expert Advisory Group to the Department of Health, was an advisor to the HSIB establishment team, and has previously held the post of Associate Director of Research and Evaluation ('researcher-in-residence') at HSIB. He also declares consultancy in patient safety for NHS and other healthcare organisations. 
Funding: Not applicable.

Ethics approval: Not applicable.

Guarantor: Carl Macrae

Acknowledgements: Views are those of the author.

Contributorship: Article the sole work of CM. 
Improving patient safety across entire healthcare systems remains an urgent and complex challenge.(1) One important strategy for system-wide safety improvement involves investigating and addressing the system-wide sources of risk that contribute to unsafe care. Common types of safety incident-such as wrong site surgery or delayed diagnosis-can harm different patients in different places at different times, but often result from very similar circumstances and underlying problems.(2) Equally, factors that contribute to unsafe care arise in many parts of the healthcare system: poor design of equipment, gaps in training, misguided regulatory incentives, inadequate funding, and much else besides.(3) The healthcare systems of England and Norway are currently experimenting with a new and ambitious approach to address system-wide sources of risk. Both have created national, independent safety investigation bodies that will investigate serious patient safety risks that span the healthcare system and develop system-wide recommendations for learning and improvement. In England, the Healthcare Safety Investigation Branch (HSIB) became operational in April 2017,(4) following proposals put forward in this journal.(5) In Norway, the State Investigation Commission for Health and Care Services (UKOM) becomes operational in 2019.(6)

Independent, system-wide and learning-focused safety investigation bodies like these have long and successful histories in other safety-critical sectors such as aviation and the railways.(7) But in healthcare, this is a relatively new and untested approach that faces significant challenges.(6) Some of these challenges are social and cultural, such as building trust and maintaining independence.(8) But many of the most immediate challenges are more practical and tangible: developing systems-focused investigation methods and safety analysis tools; establishing new approaches to designing impactful, influential and system-wide recommendations; and building new infrastructure to share findings and knowledge in ways that support the translation of recommendations into action. In addressing these challenges, these new 
investigation bodies have a unique opportunity to have a broad and meaningful impact on system-wide safety, and to fundamentally reformulate how safety investigations are conducted across healthcare. To do this, five core strategies would seem particularly important in guiding the development of these new investigative organisations (Table 1). These strategies translate the fundamental principles of national, system-wide safety investigation into more concrete objectives for strategic development, $(5,6,7)$ and draw on both early experiences in the English health system combined with insights from other learning-oriented, systems-focused investigative practices.(8)

\section{Untangle systemic risks: explain the sources of system-wide safety problems}

National safety investigation bodies need to relentlessly examine the serious, systemspanning risks that no other organisation is in a position to fully grapple with. Many safety issues are challenging because they emerge from the collective actions of many different healthcare actors-such as healthcare providers, regulators, funders and manufacturers-but cannot be solved by any single one.(10) The underlying sources of system-wide risks can be perceived by many organisations as simply 'too big' to tackle on their own. This includes system-wide issues such as poor staffing levels and gaps in workforce planning; inappropriately designed and poorly integrated technology and equipment; and the disruptive impacts of organisational restructures and service reorganisations. The systemic sources of risk in healthcare are rarely subjected to practical, regular and dispassionate investigation-and that is precisely what these new safety investigation bodies must do. Their independent status and system-wide remit give them both the ability and the responsibility to regularly and unflinchingly reveal how serious safety issues at the 'sharp end' of care are driven by 
complex, system-wide problems at the 'blunt end'.(5) To do this effectively, national investigation bodies need to strike a careful balance. They need to identify tangible, concrete safety problems that either have caused-or have the potential to causeconsiderable harm to patients, and carefully trace the factors throughout the healthcare system that produce and contribute to those problems. But they also need to avoid casting the net too wide(11) and becoming overwhelmed by the scale and quantity of safety issues that exist across healthcare. Investigative attention needs to focus on the factors that present the greatest risk to the safety of future patients, and that no single organisation can easily address on its own. National healthcare investigation bodies need clear criteria not only for prioritising which risks to investigate to start with, but also to determine which systemic safety factors should be focused on as investigations unfold.

\section{Reconfigure systems: construct innovative and co-creative recommendations}

National safety investigation bodies need to focus on developing robust recommendations that seek to fundamentally reengineer key parts of the patient safety landscape. Systematic efforts to manage patient safety are barely two decades old and safety systems remain relatively under-developed in many areas of healthcare.(12) Improving system-wide safety therefore requires much more than issuing recommendations that simply tinker with one small piece of existing policy or practice at a time. Tackling system-wide risks requires system-wide work. Investigation bodies have unique powers to issue recommendations to any and all actors across the healthcare system.(6,9) They need to use those powers to bring together diverse experts and organisations into new coalitions capable of 
reconfiguring key aspects of the healthcare system. Safety recommendations must be carefully crafted to set ambitious goals, align the motivations of different stakeholders and foster collaborative work. Initially, this will likely involve recommendations that target the creation or reconfiguration of basic building-blocks of patient safety infrastructure, such as new systems for data collection, data sharing and safety management in specific parts of healthcare. These fundamental components will need to be targeted because many aspects of healthcare safety systems remain at a relatively early stage of maturity. National investigators in other industries do the same-as illustrated by the foundational recommendations made to the autonomous vehicle industry in response to recent failures. $(13,14)$ Developing systemic safety recommendations of this nature will be complex and challenging. To help ensure such safety recommendations have an impact-and to understand and learn when they do not-requirements to commission systematic, independent evaluation should be built in to all bundles of safety recommendations. National safety investigation bodies also need to conduct their own regular evaluations of the impacts of their safety recommendations, and where necessary investigate instances where the health system has struggled to learn and continues to expose patients to unacceptable risk.

\section{Show your working: develop methodology and share ongoing evaluation}

National safety investigation bodies need to be visible and active leaders in the development and application of rigorous safety investigation and analysis methods. The analytical demands of conducting national, system-wide safety investigations that span entire healthcare systems are considerable. Investigations will need to encompass everything from individual cognition to the design of regulatory regimes. 
Many safety analysis tools and investigation models exist,(15) some specifically applied to healthcare.(16,17) But the diversity and complexity of healthcare means that a broad and pragmatic methodological approach will be needed. National investigation bodies will need to identify, test, adapt and evaluate a range of methods to build a toolbox of approaches that can be flexibly applied to different problems in diverse settings. Existing methods and tools will need to be carefully adapted to fit the different contexts of healthcare, and to ensure that they are practical to use and straightforward to explain. Moreover, it is essential that this methodological toolbox is developed and evaluated in public-both to widely share investigative tools and examples of good practice, and to build trust and establish the legitimacy of investigative findings and recommendations. The activities and outputs of investigations themselves will also need to be carefully and thoroughly evaluated, to support ongoing improvement and to model the rigour and honesty that these investigation bodies seek to encourage in the wider system.

\section{Narrative and voice: reveal the complexity of practice and experience}

National safety investigation bodies need to be at the forefront of explaining the experiences of patients, families-and staff-impacted by safety events and the practical challenges of delivery safe healthcare. Harm can accumulate and evolve over time throughout patient journeys, $(12,18)$ and many critical aspects of healthcare practice can remain invisible to those overseeing healthcare systems.(19) Patients and families are often the only people who experience the full trajectory of care and harm,(20) and frontline staff are often the only people who understand the full demands of practical work. All this hard-won knowledge is essential to improving patient safety, but is not always well used.(21) National safety investigation bodies 
are uniquely placed to give voice to the sometimes ignored patients, families and practitioners affected by safety issues, legitimising this practical knowledge and producing authoritative narratives that explain both the experience and emergence of harm. Investigations should sensitively explore the reflections and aspirations of those affected, produce accounts of safety events from multiple viewpoints, document the gaps between practical work and the expectations and policies that seek to guide it,(22) and experiment with other ways of honouring the practical wisdom of those closest to events.

\section{Make risks visible: generate active responsibility and practical knowledge}

National safety investigation bodies need to consistently generate new knowledge of systemic risk, draw widespread attention to those risks and create public accountability for addressing them. Practical information on specific sources of risk in healthcare systems is not always widely accessible.(23) In other safety-critical industries, accident investigators routinely publish detailed investigations that document the most serious safety problems, building an ever-growing open repository of practical knowledge on the nature of risk. National investigation bodies in healthcare need to do the same. They also need to regularly and forcefully direct attention to the patient safety problems that they identify by supplementing technical reports with engaging films and other compelling media; $(7,24,25)$ highlighting crosscutting safety issues and "most wanted" or "watch lists" such as those published by investigators in other industries; $(26,27)$ and visualising the risk landscape to map past and ongoing investigations and build a public picture of safety priorities. Ultimately, national investigation bodies will need to create new forms of public accountability for safety improvement, and maintain a public record of safety 
recommendations-alongside subsequent responses, actions and commentary-to provide a mechanism for generating "active responsibility" for improving safety in the future.(28)

\section{An experiment in improving investigation}

The establishment of independent, system-spanning safety investigation bodies in England and Norway is a watershed moment for patient safety. But this experiment in health policy remains at an early stage and faces many practical challenges, each of which needs to be addressed carefully and thoughtfully. These new organisations have a deep responsibility to the patients, families, practitioners and policymakers who worked hard to bring them into existence. They must now develop robust investigation methods, model a culture of openness and learning, work tirelessly and intelligently to drive systemic change-and ultimately speak truth to power. 


\section{References}

1. Shekelle PG, Pronovost PJ, Wachter RM, McDonald KM, Schoelles K, Dy SM, et al. The Top Patient Safety Strategies That Can Be Encouraged for Adoption Now. Ann Intern Med;158:365-368.

2. Care Quality Commission. Opening the door to change: NHS safety culture and the need for transformation. London: Care Quality Commission, 2018.

3. Francis R. Report of the Mid Staffordshire NHS Foundation Trust Public Inquiry. London: The Stationery Office, 2013.

4. Department of Health. Learning not blaming. London: Department of Health, 2015.

5. Macrae C, Vincent C. Learning from failure: the need for independent safety investigation in healthcare, $J R$ Soc Med 2014; 107(11) 439-443.

6. Wiig S, Macrae C. Introducing national healthcare safety investigation bodies, Br J Surg 2018;105:1710-1712.

7. Macrae $\mathrm{C}$ and Vincent C. Investigating for Improvement. Building a National Safety Investigator for Healthcare. Clinical Human Factors Group Thought Paper, 2017, https://chfg.org/investigating-for-improvementbuilding-a-national-safety-investigator-for-healthcare/ (accessed March 2019)

8. Macrae C. Close Calls: Managing Risk and Resilience in Airline Flight Safety. London: Palgrave, 2014.

9. Macrae C, Vincent C. A new national safety investigator for healthcare: the road ahead. $J R$ Soc Med 2017; 110: 90-92.

10. Dixon-Woods M, Pronovost PJ, Patient safety and the problem of many hands, BMJ Qual Saf 2016;25:485-488.

11. Reason JT. Are we casting the net too wide in our search for the factors contributing to errors and accidents? In: Misumi J, Wilpert B, Miller R eds. Nuclear Safety: An Ergonomics Perspective. Boca Raton: CRC Press 1999:212223. 
12. Vincent C, Amalberti R. Safer healthcare. Strategies for the real world. London: Springer; 2016.

13. National Transportation Safety Board. Collision Between a Car Operating With Automated Vehicle Control Systems and a Tractor-Semitrailer Truck Near Williston, Florida, May 7, 2016. Washington, DC: NTSB, 2016.

14. National Transportation Safety Board. Preliminary report for Crash Involving Pedestrian, Uber Technologies, Inc., Test Vehicle, March 18, 2018. Washington, DC: NTSB, 2018.

15. Filho APG, Jun GJ, Waterson P, Four studies, two methods, one accident - An examination of the reliability and validity of Accimap and STAMP for accident analysis. Safety Science 2018;113:310-317.

16. Duchscherer, C and Davies, JM. Systematic Systems Analysis: A Practical Approach to Patient Safety Reviews. Health Quality Council of Alberta: Alberta, 2012.

17. Vincent C, Taylor-Adams S, Chapman EJ, Hewett D, Prior S, Strange P, Tizzard A. How to investigate and analyse clinical incidents: Clinical Risk Unit and Association of Litigation and Risk Management protocol. BMJ 2000;320(7237):777-781.

18. Vincent C, Carthey J, Macrae C, Amalberti R, Safety analysis over time: seven major changes to adverse event investigation, Imp Sci, 2017;12:151

19. Allen D, The Invisible Work of Nurses: Hospitals, Organisation and Healthcare. London: Routledge, 2014.

20. O'Hara JK, Aase K, Waring J, Scaffolding our systems? Patients and families 'reaching in' as a source of healthcare resilience. BMJ Qual Saf 2019;28:3-6.

21. Donaldson, LJ. The Wisdom of Patients and Families: Ignore it at our Peril BMJ Quality and Safety, 2015; 24: 603-604.

22. Moppett, I. K. and Shorrock, S. T. (2018), Working out wrong-side blocks. Anaesthesia, 73: 407-420.

23. Macrae C. Early warnings, weak signals and learning from healthcare disasters. BMJ Qual Saf 2014;23:440-445. 
24. Jun T, Systems Thinking: A new direction in healthcare incident investigation, https://www.youtube.com/watch?v=50YV3DqeoA8 (accessed March 2019)

25. The Human Factor: Learning from Gina's Story. See https://www.youtube.com/watch?v=IJfoLvLLoFo (accessed March 2019). 26. Transportation Safety Board of Canada, Watchlist 2018, http://www.bsttsb.gc.ca/eng/surveillance-watchlist/index.asp (accessed Jan 2019)

27. National Transportation Safety Board, 2017-208 Most Wanted List. https://www.ntsb.gov/safety/mwl/Pages/default.aspx (accessed Jan 2019) 28. Braithwaite $J$, The essence of responsive regulation, UBC L Rev 2011;44(3):475-520. 